\title{
Na-like Autoionizing Levels: Plasma Diagnostics and Prospects for Photopumped Soft X-Ray Lasers
}

\author{
A. L. Osterheld, J. Dunn, B.K.F. Young, S.B. Libby, A. Szoke, R.S. \\ Walling, W.H. Goldstein and R.E. Stewart \\ Lawrence Livermore National Laboratory, L-59 \\ P.O. Box 808, Livermore, CA 94550
}

\author{
A. Ya. Faenov, I. Yu. Skobelev and S. Ya. Khakhalin \\ Multicharged Ions Spectral Data Center, National Scientific-Research Institute for Physical- \\ Technical and Radio-Technical Measurements (VNIIFTRI) \\ Mendeleevo, Moscow Region, 141570 Russia
}

\begin{abstract}
We have investigated the kinetics of doubly excited sodium-like copper states with $313 l^{\prime}$ and $314 l^{\prime}$ configurations, and the feasibility of photopumped $x$-ray lasing schemes and plasma diagnostics using transitions from these levels. These $x-$ ray lasing schemes, like similar lasing schemes in Li-like ions (1-3), have high quantum efficiency and potentially reduced radiative trapping effects. In addition, the satellite transitions emitted by these sodium-like levels may provide valuable diagnostics of $x$-ray laser plasmas.
\end{abstract}

\section{INTRODUCTION}

Sodium-like autoionizing levels have interesting applications in $\mathrm{x}$-ray laser research. The $n=3-2$ and $n=4-2$ satellite spectra from these levels provide valuable information about the electron temperature, density and ionization distribution. In addition, transitions between these levels may be inverted, if excited by resonant photopumping. These schemes are analogous to proposed lithium-like photopumped $x$-ray lasing schemes (1-3).

While the plasma conditions in neon-like $\mathrm{x}$-ray laser plasmas have been diagnosed by seeding a low- $Z$ tracer into the plasma (4), it may be advantageous to use the $\mathrm{L}$-shell radiation produced by the lasing element. The use of $n=3-2$ sodium-like satellite transitions for plasma diagnostics has previously been investigated $(5-7)$. The $n=4-2$ satellite transitions offer several advantages: a) the lower optical depth of the corresponding neon-like resonance lines, b) the better isolation of the 4-2 satellites, and c) the smaller contributions from MASTER resonance excitation and cascades to the resonance and satellite lines. Because 


\section{DISCLAIMER}

This report was prepared as an account of work sponsored by an agency of the United States Government. Neither the United States Government nor any agency thereof, nor any of their employees, make any warranty, express or implied, or assumes any legal liability or responsibility for the accuracy, completeness, or usefulness of any information, apparatus, product, or process disclosed, or represents that its use would not infringe privately owned rights. Reference herein to any specific commercial product, process, or service by trade name, trademark, manufacturer, or otherwise does not necessarily constitute or imply its endorsement, recommendation, or favoring by the United States Government or any agency thereof. The views and opinions of authors expressed herein do not necessarily state or reflect those of the United States Government or any agency thereof. 


\section{DISCLAIMER}

Portions of this document may be illegible in electronic image products. Images are produced from the best available original document. 


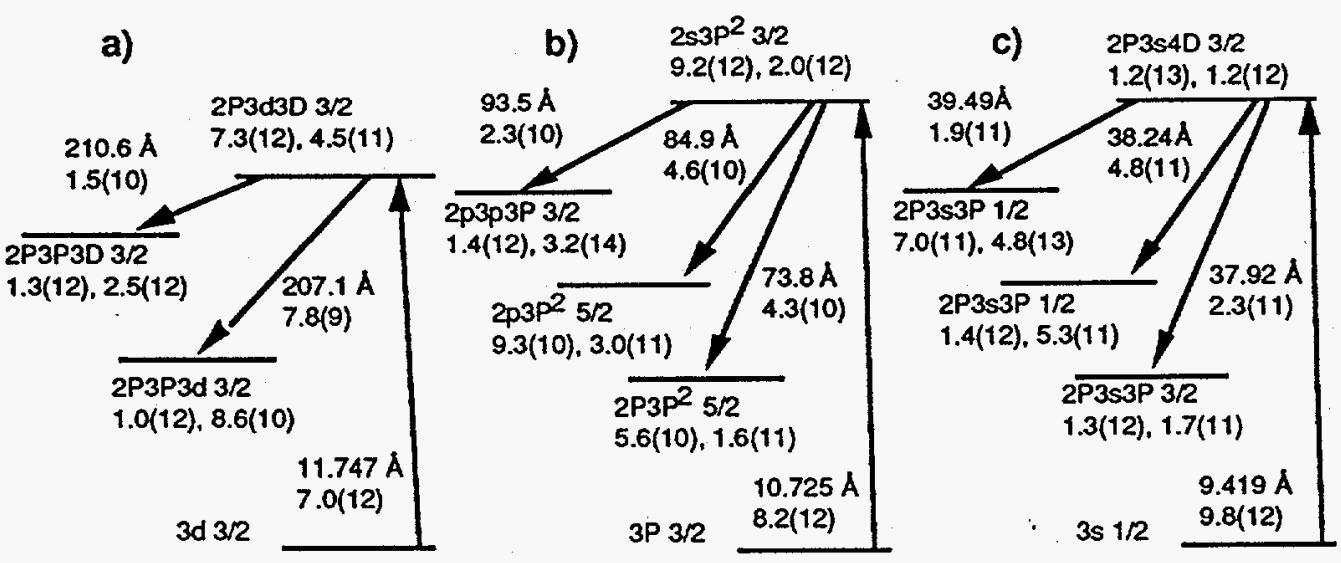

FIGURE 1. Energy level diagrams of the important levels for examples of a) $n=3-3$, b) inner shell $2 s-2 p$, and c) $n=4-3$ Na-like copper photopumped $x$-ray lasing schemes.

of lack of space, we refer the reader to references 8 and 9 for a discussion of the application of $n=4-2$ satellite lines to plasma diagnostics.

Resonantly photopumped $\mathrm{x}$-ray laser schemes between autoionizing levels potentially have high quantum efficiency and reduced trapping problems. In most conventional lasing schemes, the lower laser level is depleted by fast radiative decays to low-lying ground levels. As the transverse size of the $\mathrm{x}$-ray laser is increased, the reabsorption of this radiation increases the effective lifetime of the lower level, reducing the inversion density. The conventional collisional $x$-ray lasers have low quantum efficiency, as they lase on $\Delta n=0$ transitions between highly excited levels. This is because the levels which are strongly excited by electron collisions typically do not have $\Delta n>0$ decays to levels with shorter lifetimes. The use of transitions between autoionizing levels opens up the possibility of lasing transitions whose lower levels decay nonradiatively. This potentially ameliorates the trapping problem, and allows $\Delta n>0$ lasing transitions.

\section{THEORETICAL MODEL AND RESULTS}

We investigate the kinetics of the autoionizing levels using a collisionalradiative model. The level populations are determined by solving a set of linear rate equations which describe the various excitation, de-excitation, ionization and recombination processes which transfer population between the energy levels. The collisional-radiative model consists of detailed atomic data embedded in an average-level model. Further details may be found in ref. 10 .

The detailed atomic data come from the Hebrew University-Lawrence Livermore Atomic Code package (HULLAC). This code is used to calculate wave functions, energy levels, autoionization rates, and all important radiative transition rates from the relativistic, multi-configuration parametric potential model with full configuration interaction (11-13). Relativistic, distorted wave electron excitation cross-sections are calculated for all transitions (14), including all collisions between doubly excited states. The cross-section calculations use a powerful angular factorization and an empirically motivated interpolation scheme for calculating collisional rates. This code package uniquely combines 



FIGURE 2. Gain calculations for the a) $n=3-3$, and b) $n=4-3$ sodium-like copper photopumped $x$-ray lasing schemes.

accuracy and highly efficient computational techniques and enables practical calculations of even very complex atomic systems.

In the present work the collisional-radiative model explicitly includes the doubly excited sodium-like levels with configurations $2 s^{2} 2 p^{5} 3131^{\prime}$ and $2 \mathrm{~s}^{1} 2 \mathrm{p}^{6} 313 \mathrm{l}^{\prime} l, l^{\prime}=0-2$, and $2 \mathrm{~s}^{2} 2 \mathrm{p}^{5} 314 \mathrm{l}^{\prime}$ and $2 \mathrm{~s}^{1} 2 \mathrm{p}^{6} 3 \mathrm{l}^{4} \mathrm{l}^{\prime} l=0-2, l^{\prime}=0-3$. There are slightly more than 1000 of these levels. This full treatment of the kinetics consistently describes the mechanisms which populate the doubly excited states and allows the gain calculations to include the effects of cascades between autoionizing levels, collisional quenching, and collisional broadening.

The main goal of the present work is to investigate the kinetics to identify promising lasing schemes in a single ion (copper), rather than to search for resonant line pairs in sodium-like ions of many elements. The large number of sodium-like autoionizing levels gives rise to many potential lasing transitions, most of which can not be pumped to practical inversion densities. Initially, an analytic three level formula was used to select transitions for further study with the full collisional-radiative model.

Because of the complex level structure of a many-electron ion, the photopumped sodium-like lasing schemes are more varied than the corresponding lithium-like case. The sodium-like lasing schemes fall into three qualitatively different classes. These include $\Delta n=0, n=3-3$ (and $n=4-4$ ) lasing transitions, inner shell $2 s-2 p$ transitions pumped by $2 s-3 p$ (or $2 s-4 p$ ) excitations, and $n=4-3$ transitions produced by pumping the $3141^{\prime}$ doubly excited levels. The $n=3-3$ schemes lase at wavelengths similar to the normal neon-like copper $x$-ray lasers, but will be the easiest to achieve experimentally. The inner-shell $2 s-2 p$ schemes have no analogy in lithium-like ions. These are at relatively short wavelength even in mid- $Z$ ions, and are analogous to collisional (14) and photopumped (15) neon-like $2 \mathrm{~s}-2 \mathrm{p}$ lasing schemes previously investigated. The $\mathrm{n}=4-3$ schemes are directly analogous to the lithium-like schemes, and are at quite short wavelength. A favorable example of each type of lasing scheme is discussed below.

Examples of each type of lasing scheme are shown in Figure 1, along with the relevant lifetimes and transition rates. The levels are labeled with their total angular momentum and a relativistic configuration name formed from the orbitals of the $n=2$ hole and the outer two electrons (where $2 p=2 p_{1 / 2}, 2 P=2 p_{3} / 2$, 

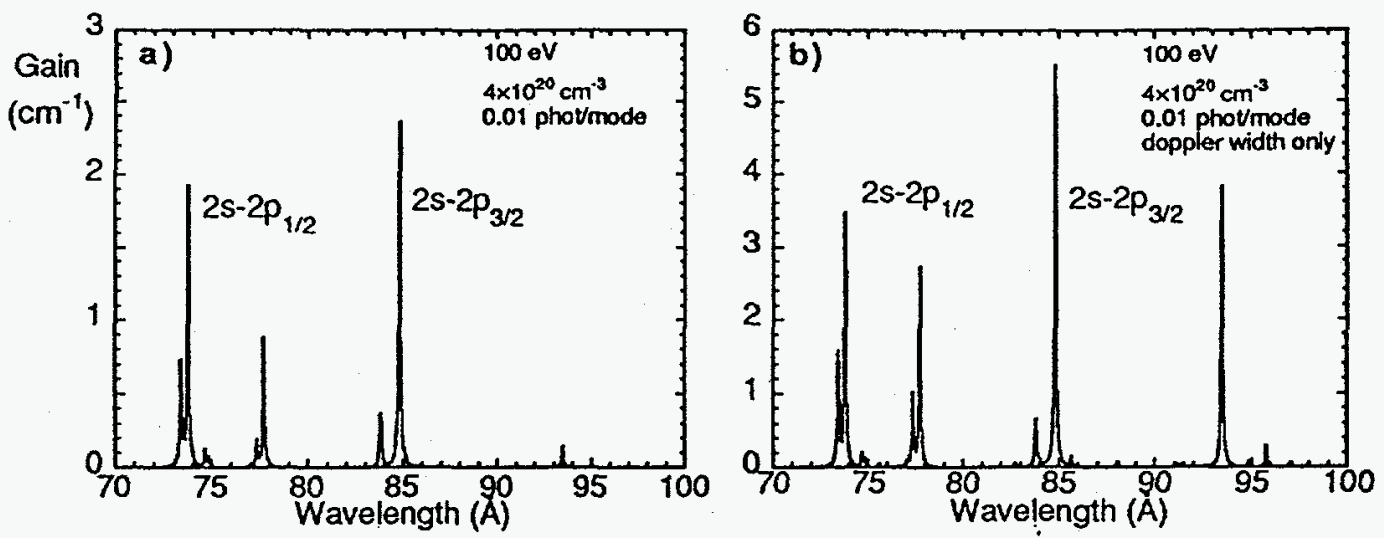

FIGURE 3. Gain calculations for the $2 s-2 p$ lasing scheme a) including autoionization and collisional widths, and b) with Doppler broadening only.

etc.). The total radiative and autoionization decays rates ( $\left.\sec ^{-1}\right)$ are also indicated, as well as transition wavelengths and rates, where $1.5(10)$ is shorthand for $1.5 \times 10^{10}$.

Steady state gain calculations are shown in Figs. 2 and 3. The calculations are for electron and ion temperatures of 100 and $75 \mathrm{eV}$, and an electron density of $4 \times 10^{20} \mathrm{~cm}^{-3}$. For these initial calculations, the ionization balance was not calculated consistently with the kinetics, and the total abundance of sodium-like ions was taken to be $50 \%$. In each case, the photopumped line was pumped with an intensity of 0.01 photons/mode. This corresponds to equivalent radiation temperatures of roughly 228,250 , and $285 \mathrm{eV}$ for the schemes in Fig. 1 . The gain transitions in the $n=4-3$ case in Fig. $2 b$ include both $4 d-3 p$ transitions directly pumped and $4 \mathrm{f}-3 \mathrm{~d}$ gain lines produced by collisional transfer. This effect is most prominent for this case because of the strong $3 \mathrm{~d}-4 \mathrm{f}$ collision rates. The gains in all cases are affected by decreased lifetimes owing to fast autoionizations or collisional mixing. This is illustrated in Fig. 3, which compares calculated $2 \mathrm{~s}-2 \mathrm{p}$ gains including the auger and collisional lifetimes to values calculated from Doppler broadening alone. In particular, the line at 93.5 $\AA$ is drastically reduced owing to the rapid auger decay of the lower level (see Fig. 1b). Even larger lifetime effects occur for the longer wavelength $n=3-3$ gain transitions. The gains in Fig. $2 \mathrm{a}$ would increase by a factor of four if only Doppler broadening were considered.

\section{CONCLUSIONS}

We have investigated the prospects for soft $x$-ray lasing between autoionizing sodium-like copper levels pumped by photo-resonant excitation and have identified a number of promising gain transitions on $\Delta n=0$ and $\Delta n=1$ transitions, including relatively short wavelength transitions in medium- $Z$ ions. The potential lasing transitions fall into three categories. These include long wavelength $n=3-3$ transitions which are the easiest to realize experimentally; inner-shell $2 s-2 p$ transitions which are much shorter in wavelength than the normal neon-like lasing transitions; and $\Delta n=1, n=4-3$ transitions which are at short wavelengths, but will be very difficult to experimentally achieve. 
A number of general conclusions may be drawn. All of these schemes require fairly intense pumping transitions. This is because the existence of multiple sodium-like ground states reduces the ion density in any given ground level of a three-level scheme, and because the lifetimes of the highly excited upper lasing states are not truly "metastable". In addition, the gain coefficients are strongly reduced by both the homogenous broadening from autoionization and collisional decays, and the quenching of the inversions from collisional mixing. Finally, these photopumped lasing schemes require separate pump and lasing plasmas. This results from the conflicting requirements of maintaining a large population of sodium-like ions and simultaneously producing an intense pump line whose transition energy is large compared to the sodium-like ionization potential. This requirement of separate plasmas reduces the application of this method for aperture scaling. The amelioration of the trapping problem is offset by the problem of propagating the externally produced pumping radiation into the lasing region.

\section{ACKNOWLEDGMENTS}

Work performed under the auspices of the U.S. Dept. of Energy by the Lawrence Livermore National Laboratory under Contract No. W-7405-Eng-48.

\section{REFERENCES}

1. Elton, R.C., NRL Report 9103 (1988); Elton, R.C., X-ray Lasers, New York: Academic Press, 1990.

2. Lunney, J.G., Optics Comm. 53, 235 (1985).

3. Libby, S.B., Osterheld, A.L., Szoke, A., Walling, R.S., Young, B.K.F., "Progress toward Xray lasing between autoionizing transitions, in Proceedings of the 3 rd International Conference on X-Ray Lasers, IOP Conference Series 125, 1992, pp. 163-166.

4. Young, B.K.F., Osterheld, A.L. Shimkaveg, G.M., Shepherd, R.L., Walling, R.S., Goldstein, W.H., and Stewart, R.E., J.Q.S.R.T. 51, 417 (1994).

5. Goldstein, W.H., Walling, R.S., Bailey, J., et al., Phys. Rev. Lett. 58, 2300 (1987).

6. Peyrusse, O., Combis, P., Louis-Jacquet, M., et. al., J. Appl. Phys. 65, 3802 (1989).

7. Khakhalin, S.Ya., Bryunetkin, B.A., Skobelev, I.Yu., Faenov, A.Ya., Nilsen, J., Osterheld, A., Pikuz, S.A., JETP 78, 633 (1994), and references therein.

8. Osterheld, A.L., Dunn, J., Young, B.K.F., Goldstein, W.H., Faenov, A. Ya., Skobelev, I. Yu., Khakhalin, S. Ya., submitted to Phys. Rev. A, (1994).

9. Khakhalin S.Ya., Dyakin, V.M., Faenov, A.Ya., Fiedorowicz, H., Bartnik, A., Parys, P., Nilsen, J., and Osterheld, A., submitted to JOSA B, (1994).

10. Osterheld, A.L., Walling, R.S., Young, B.K.F., Goldstein, W.H., Shimkaveg, G., MacGowan, B.J., DaSilva, L., London, R., Matthews, D. and Stewart, R.E., J. Quant. Spectrosc. Radiat. Transfer, 51, 263 (1994).

11. Klapisch, M., Comput. Phys. Commun. 2, 239 (1971).

12. Klapisch, M., Schwob, J.L., Fraenkel, B.S., and Oreg, J., J. Opt. Soc. Am. 61, 148 (1977).

13. Oreg, J., Goldstein, W.H., Klapisch, K., Bar-Shalom, A., Phys. Rev. A 44, 1750 (1991).

14. Bar-Shalom, A., Klapisch, M. and Oreg, J., Phys. Rev. A 38, 1773 (1988).

15. Enright, G.D., Baldis, H.A., Dunn, J., La Fontaine, B. and Villeneuve, D.M., "A search for gain on 2p-2s transitions in a collisionally excited Ge plasma" in Proc. OSA Conf. on Short Wavelength Coherent Radiation: Generation and Application 11, 87 (1991).

16. Politov, V.Yu., Loboda, P.A., Lykov, V.A. and Nilsen, J., Optics Comm 108, 283 (1994). 